\title{
Non-small-cell lung cancer metastasis to the oral cavity: A case report
}

\author{
HIROYUKI ITO ${ }^{1}$, KOJIRO ONIZAWA ${ }^{1}$ and HIROAKI SATOH ${ }^{2}$ \\ ${ }^{1}$ Division of Oral and Maxillofacial Surgery and ${ }^{2}$ Division of Respiratory Medicine, \\ Mito Medical Center, University of Tsukuba, Mito, Ibaraki 310-0015, Japan
}

Received April 19, 2016; Accepted May 30, 2016

DOI: $10.3892 / \mathrm{mco} .2017 .1141$

\begin{abstract}
We herein report an unusual occurrence of lung cancer metastasis to the oral cavity, reflecting the progression of the primary malignancy. A biopsy of oral lesions should always be performed, even of those located in uncommon sites, in patients with a history of uncontrolled lung cancer. Although this is a rare occurrence, our patient exhibited characteristics suggestive of oral metastasis from lung cancer, namely presence of lung adenocarcinoma, development of oral metastasis during the clinical course of the disease, accompanying multiple metastatic lesions, including to the brain and abdomen, and rapid fatal progression after the manifestation of oral metastasis. It is important to acknowledge the possibility of metastasis to the oral cavity in lung cancer patients with extensive metastases. Prompt diagnosis by biopsy of the lesion is required to establish the correct diagnosis.
\end{abstract}

\section{Introduction}

Metastasis to the oral cavity is a rare event in cancer patients. This distant spread indicates that cancer cells may reach several sites throughout the body via the bloodstream and the lymphatic system $(1,2)$. Similar to other malignant diseases, oral metastasis from lung cancer reflects the progression of the primary malignancy, and the majority of such patients present with multiple metastatic sites $(3,4)$. Recently, however, some researchers reported patients with no metastasis other than to the oral cavity (5-7). The clinical presentation of oral metastasis may vary from local swelling or pain to paresthesia (2). Oral metastatic lesions are divided into bone (mandible and maxilla) and mucosal metastases (1). However, as in the majority of the cases both the bone and the mucosa are affected, the first metastatic site may not always be apparent. We herein report a case with oral metastasis from lung adenocarcinoma and

Correspondence to: Dr Hiroaki Satoh, Division of Respiratory Medicine, Mito Medical Center, University of Tsukuba, 3-2-7 Miya-machi, Mito, Ibaraki 310-0015, Japan

E-mail: hirosato@md.tsukuba.ac.jp

Key words: metastasis, lung adenocarcinoma, oral cavity review previously reported cases of lung cancer patients with oral metastasis.

\section{Case report}

An 85-year-old man presented with a right-sided buccal tumor. One year prior, the patient had been diagnosed with primary lung adenocarcinoma. As the performance status was poor, the patient only received supportive care. The self-reported history of the buccal lesion was rapid growth of the mass over a 2-week period with associated bleeding and pain. The patient was referred to the Oral and Maxillofacial Surgery Unit of the Mito Medical Center (Mito, Japan) for investigation and management. Physical examination revealed a marked swelling on the left upper buccal gingiva (Fig. 1). T2-weighted magnetic resonance imaging also revealed a marked swelling on the left upper buccal gingiva (Fig. 2). A biopsy specimen of the lesion obtained under local anesthesia without complications was sent for histopathological examination. The histopathology of the specimen was morphologically consistent with adenocarcinoma and immunohistochemical staining was positive for cytokeratin-7 and thyroid transcription factor-1, which supported the diagnosis of metastasis to the oral cavity from lung adenocarcinoma. Brain magnetic resonance imaging and chest and abdominal computed tomography scans revealed metastatic lesions in the brain, pancreas, adrenal glands and kidneys. The oral lesion was irradiated, but the response was poor and the patient soon succumbed due to cachexia. Informed consent was obtained from the patient's family for the publication of the case details.

\section{Discussion}

Metastasis to the oral cavity is rare and represents $\sim 1-2 \%$ of all oral malignancies $(1,8)$. Due to its rarity and lack of specific gross characteristics, oral metastasis may be mistakenly diagnosed as a benign primary oral disease at the time of detection. Some researchers reported that the most frequent malignancies metastasizing to the oral cavity are lung, breast, kidney, prostate, thyroid, and stomach cancers (8-10), whereas others describe lung cancer as the most common malignancy metastasizing to the oral cavity, followed by breast, kidney and liver cancers $(11,12)$. Metastasis to the oral cavity may occur at any age, but is most common during the fifth and sixth decades 


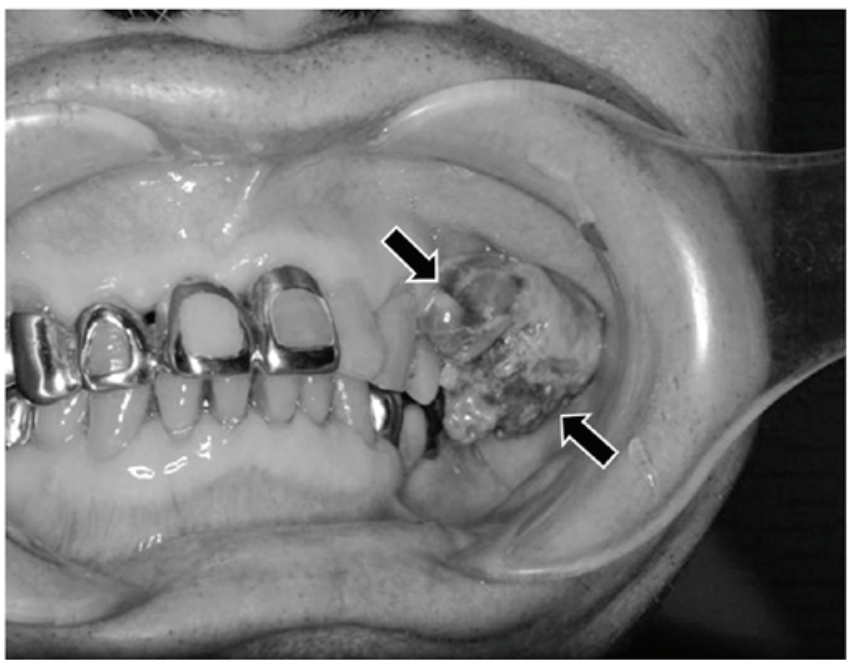

Figure 1. Physical examination revealed a marked swelling on the left upper buccal gingiva.

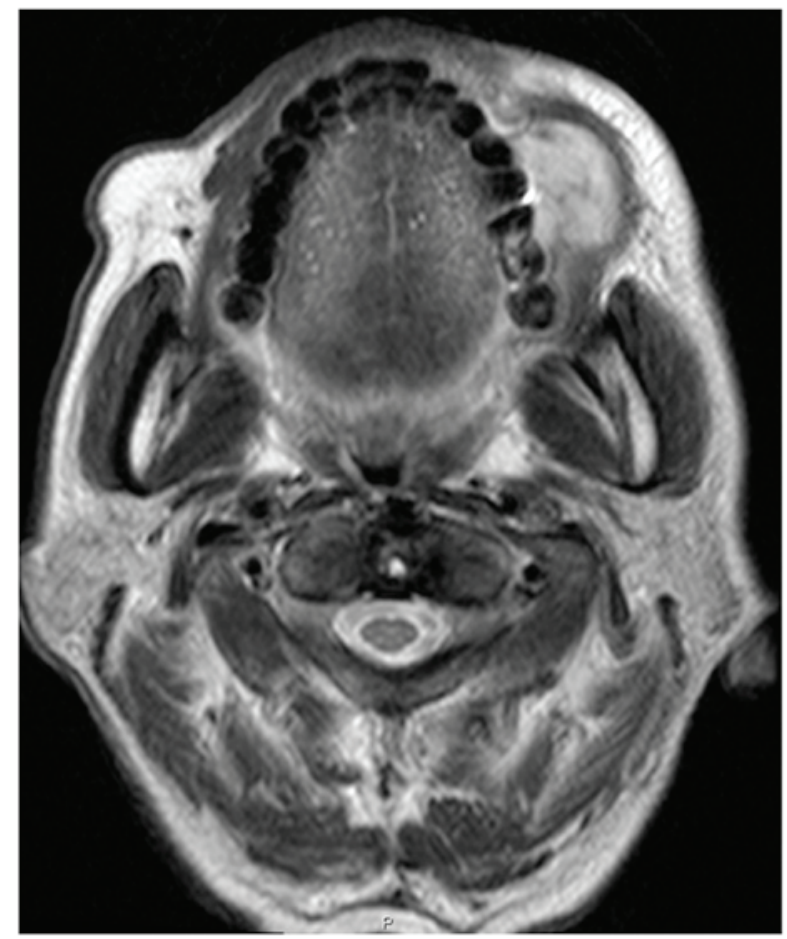

Figure 2. T2-weighted magnetic resonance imaging showing a mass located in the left upper buccal gingiva.

of life (8). Hischberg et al reported that $70 \%$ of oral metastases manifested after the primary tumor became evident, while the remaining $30 \%$ were the first clinical manifestation of primary tumor spread (13). The patient in the present case was elderly and the oral metastasis was identified 1 year after the primary lung cancer was diagnosed.

A rapidly progressing lesion accompanied by pain and paresthesia are the most common symptoms, whereas a bony swelling with tenderness over the affected area is observed in several cases (14). Our patient complained of a non-healing mass on the surface of his left upper buccal gingiva. The lesion was first considered to be a primary oral disease; however, excisional biopsy revealed lung adenocarcinoma.
Of note, the majority of oral metastases are located in the mandible, with a maxillary location being less common. Possible routes of metastasis to the oral cavity include the bloodstream and the lymphatic system. It is generally accepted that the mandible and the maxilla do not contain lymphatic vessels; therefore, the only practical route for metastasis to the mandible is the blood vessels (15). Metastatic foci in the bones are mainly situated in the red marrow (16); however, the mandible in adults contains red marrow mainly in the area of the ascending ramus and in the angles, whereas the maxilla only contains fatty marrow. This may explain the predilection of the mandible to distant metastases, as well as the preferred localization in the maxilla (17). However, direct buccal or gingival metastases from lung cancer, to sites other than the maxilla and mandible, have been reported (1). In our case, as oral metastasis extended from the left upper buccal gingiva to the left maxilla, the first metastatic site was not apparent.

The occurrence of metastasis to the oral cavity is generally a sign of extensive metastases, indicating a fatal outcome shortly after the diagnosis of oral metastasis $(10,13)$. Our patient had extensive metastases when the oral lesion was identified. This distant spread indicates that cancer cells may reach numerous sites in the entire body via the bloodstream and the lymphatic system. Of note, as observed in our patient, the majority of patients with oral cavity metastasis from a primary cancer share 4 characteristics: i) Lung adenocarcinoma, ii) development of oral metastasis during the clinical course of the disease, iii) accompanying multiple metastatic lesions, including to the brain and abdomen, and iv) rapid fatal progression after the manifestation of oral metastasis $(3,5,7,18-20)$.

When a new oral lesion is identified, either by the physician or the patient, in patients who have a history of uncontrolled lung cancer, a biopsy of the lesion should be performed, even in cases with an uncommon location. It is important to acknowledge the possibility that metastasis to the oral cavity may occur in lung cancer patients with extensive metastases. Prompt diagnosis may lead to beneficial treatment in certain patients, particularly those harboring epidermal growth factor receptor mutations $(21,22)$, and an improved quality of life. However, adequative palliative care to improve the quality of life and prolong survival is the primary goal for patients with metastasis to the oral cavity, until more efficient treatments are developed.

\section{References}

1. Hirshberg A, Shnaiderman-Shapiro A, Kaplan I and Berger R: Metastatic tumours to the oral cavity-pathogenesis and analysis of 673 cases. Oral Oncol 44: 743-752, 2008.

2. van der Waal RI, Buter J and van der Waal I: Oral metastases: Report of 24 cases. Br J Oral Maxillofac Surg 41: 3-6, 2003.

3. Olsen JB, Sim F and Chandu A: Non-small cell lung cancer metastasis to the oral cavity: A case report. Aust Dent J 59: 520-524, 2014.

4. Curien R, Moizan H and Gerard E: Gingival metastasis of a bronchogenic adenocarcinoma: Report of a case. Oral Surg Oral Med Oral Pathol Oral Radiol Endod 104: e25-e28, 2007.

5. Rajini Kanth M, Ravi Prakash A, Raghavendra Reddy Y, Sonia Bai JK and Ravindra Babu M: Metastasis of lung adenocarcinoma to the gingiva: A rare case report. Iran J Med Sci 40: 287-291, 2015.

6. Orlandi A, Basso M, Di Salvatore M, Federico F, Cassano A and Barone C: Lung adenocarcinoma presenting as a solitary gingival metastasis: A case report. J Med Case Rep 5: 202, 2011. 
7. Tatlidil R and Gözübüyük MM: Mucinous adenocarcinoma of lung presenting as oral metastases: A case report and literature review. J Endod 37: 110-113, 2011.

8. Hirsberg A and Buchner A: Metastatic tumours to the oral region. An overview. Eur J Cancer B Oral Oncol 31B: 355-360, 1995

9. Rodado C, Gonzalez J, Huguet P, Avila M and Raspall G: Lung adenocarcinoma presenting as an oral metastasis. Med Oral 2: 315-320, 1997.

10. Nihismura Y, Yakata T, Kawasaki T and Nakajima T: Metastatic tumours of the mouth and jaw. A review of the Japanese literature. J Maxillofac Surg 10: 253-257, 1982.

11. Zachariades N: Neoplasms metastatic to the mouth, jaws and surrounding tissues. J Craniomaxillofac Surg 17: 283-290, 1989.

12. Pires FR, Sagarra R, Corrêa ME, Pereira CM, Vargas PA and Lopes MA: Oral metastasis of a hepatocellular carcinoma. Oral Surg Oral Med Oral Pathol Oral Radiol Endod 97: 359-368, 2004.

13. Hirhberg A, Leibovich P and Buchner A: Metastatic tumors to the jawbones: Analysis of 390 cases. J Oral Pathol Med 23 337-341, 1994

14. Hirshberg A, Berger R, Allon I and Kaplan I: Metastatic tumors to the jaws and mouth. Head Neck Pathol 8: 463-474, 2014.

15. Yin JJ, Pollock CB and Kelly K: Mechanisms of cancer metastasis to the bone. Cell Res 15: 57-62, 2005.

16. Berrettoni BA and Carter JR: Mechanisms of cancer metastasis to bone. J Bone Joint Surg Am 68: 308-312, 1986.
17. Kricun ME: Red-yellow marrow conversion: Its effect on the location of some solitary bone lesions. Skeletal Radiol 14: 10-19, 1985.

18. Nuyen BA and Tang CG: Gingival metastasis: A case report and literature review. Perm J 20: 71-73, 2016.

19. Villa A, Mariani U and Villa F: Oral metastasis of lung cancer. A challenge for the clinician. Oral Maxillofac Surg 16: 133-135, 2012.

20. Staalsen NH and Nielsen JS: Bronchogenic metastasis to the gingiva. Oral Surg Oral Med Oral Pathol 74: 561-562, 1992.

21. Daniels AB, Miller ML, Kotecha A and Abramson DH: Uveal metastasis from nonsmall cell lung carcinoma with dramatic response to erlotinib. Retin Cases Brief Rep 4: 390-393, 2010.

22. Chang GC, Yang TY, Wang NS, Huang CM and Chiang CD: Successful treatment of multifocal bronchioloalveolar cell carcinoma with ZD1839 (Iressa) in two patients. J Formos Med Assoc 102: 407-411, 2003. 